\title{
A LAGRANGIAN RELAXATION APPROACH TO THE EDGE-WEIGHTED CLIQUE PROBLEM
}

\author{
MARCEL HUNTING, ULRICH FAIGLE, AND WALTER KERN
}

\begin{abstract}
The $b$-clique polytope $C P_{b}^{n}$ is the convex hull of the node and edge incidence vectors of all subcliques of size at most $b$ of a complete graph on $n$ nodes. Including the Boolean quadric polytope $Q P^{n}$ as a special case and being closely related to the quadratic knapsack polytope, it has received considerable attention in the literature. In particular, the max-cut problem is equivalent with optimizing a linear function over $Q P_{n}^{n}$. The problem of optimizing linear functions over $C P_{b}^{n}$ has so far been approached via heuristic combinatorial algorithms and cutting-plane methods.

We study the structure of $C P_{b}^{n}$ in further detail and present a new computational approach to the linear optimization problem based on Lucena's suggestion of integrating cutting planes into a Lagrangian relaxation of an integer programming problem. In particular, we show that the separation problem for tree inequalities becomes polynomial in our Lagrangian framework. Finally, computational results are presented.
\end{abstract}

\section{INTRODUCTION}

Consider the complete undirected graph $K_{n}=(V, E)$ on the set $V=$ $\{1, \ldots, n\}$ of nodes with edge set $E$. We assume that to every edge $e \in E$ a weight $c_{e}$ and to every node $i \in V$ a weight $d_{i}$ is assigned. The weighted maximal $b$-clique problem $\left(W C P_{b}\right)$ is to find, among all complete subgraphs with at most $b$ nodes, a subgraph (clique) for which the sum of the weights of all the nodes and edges in the subgraph is maximal.

The weighted maximal $b$-clique problem can be seen as a Boolean quadratic problem with a cardinality constraint (Mehrotra [1997]). On the other hand, $\left(W C P_{b}\right)$ generalizes the well-studied maximum clique problem, which is known to be $N P$-hard.

Applications of $\left(W C P_{b}\right)$ can be found, for example, in location theory (see Späth [1985], Kuby [1987], Erkut et al.[1990] and Ravi et al. [1994]). Other important applications of this optimization model arise in molecular biology (see Hunting [1998]).

Date: 9 December, 1998.

1991 Mathematics Subject Classification. 90C27, 90D12.

Key words and phrases. clique polytope, cut polytope, cutting plane, Boolean quadric polytope, quadratic knapsack polytope, Lagrangian relaxation. 
Exact branch-and-cut algorithms for $\left(W C P_{b}\right)$, based on linear programming have been proposed by Dijkhuizen and Faigle [1993], Park et al. [1996], Mehrotra [1997], and Macambira and De Souza [1997]. In these four papers several facet-defining inequalities are introduced which are used as cutting planes in order to improve the quality of the upper bounds. In Faigle et al. [1994] upper bounds derived from Lagrangian relaxation are proposed. The usual branch-and-cut approach can be quite successful for special types of problems (see, e.g., Barahona et al. [1989] for the max-cut problem). The general case, however, appears to be much harder. The first three of the afore-mentioned studies deal with graphs with up to 30 nodes whereas as formulated by Macambira and de Souza - "the real challenge is to solve problems with $n \geq 40$ nodes".

The weighted maximal $b$-clique polytope generalizes the Boolean quadric polytope, which has been studied extensively (see, e.g., Padberg [1989], Deza and Laurent [1992a,b], Boros and Hammer [1993], Sherali et al. [1995] and Hardin et al. [1995]). The max-cut problem on graphs (cf. Barahona and Mahjoub [1986]) can be formulated as the problem of optimizing a linear function over the Boolean quadric polytope. On the other hand, the weighted maximal $b$-clique problem is a special case of the quadratic knapsack problem. Therefore, the corresponding polytopes are closely related. Various techniques have been proposed for solving quadratic knapsack problems (see Chaillou et al. [1986], Johnson et al. [1993], Bretthauer et al. [1995] and Helmberg et al. [1996]).

In the present paper, we propose a relaxation technique that has not been applied before to $\left(W C P_{b}\right)$ or to the related problems mentioned above. Following an idea of Lucena [1992] we combine Lagrangian relaxation with the use of cutting planes. We report computational results with a branchand-cut algorithm based on this relaxation. We expect that the technique can also be fruitfully applied to the related problems.

The paper is organized as follows. In Section 2, we give an integer programming formulation for $\left(W C P_{b}\right)$ and review polyhedral results from the literature. In Section 3, we describe two new classes of facet-defining inequalities, which we will use in the (Lagrangian) branch-and-cut algorithm, namely the $i$-clique inequalities and the generalized clique inequalities. These inequalities turn out to define facets of the Boolean quadric polytope as well.

In Section 4 , we formulate a Lagrangian problem for $\left(W C_{b}\right)$ which turns out to have solutions with nice properties. The upper bound obtained in this Lagrangian relaxation often is quite good. Adding cutting planes to the objective function, however, we can obtain even stronger bounds as our computational results show.

Using cutting planes in a Lagrangian relaxation offers a considerable advantage over the standard branch-and-cut approach: Because of the special 
structure of the Lagrangian solutions, we are able to use classes of inequalities for which the separation problem is $N P$-hard in general ( $c f$. the tree inequalities in Section 4) by adding violated inequalities to the objective function of the Lagrangian relaxation without changing the structure of the Lagrangian solutions.

\section{The $b$-Clique Polytope}

We formulate the weighted maximal $b$-clique problem $\left(W C_{b}\right)$ as an integer programming problem. We introduce for every node $i \in V$ a $(0,1)$-variable $x_{i}$ and for every edge $e \in E$ a $(0,1)$-variable $y_{e}$. We think of an edge $e \in E$ as a subset of $V$ of cardinality 2. In particular, if $e=\{i, j\}$, we call $i$ and $j$ the endpoints of $e$.

Variable $x_{i}$ equals 1 if node $i$ is in the clique and 0 otherwise, and $y_{e}$ equals 1 if edge $e$ is in the clique and 0 if not. The weighted maximal $b$-clique problem can then be formulated as follows:

$$
\max \sum_{i \in V} d_{i} x_{i}+\sum_{e \in E} c_{e} y_{e}
$$

subject to the constraints

$$
\begin{array}{ll}
\sum_{i \in V} x_{i} \leq b & \\
y_{e}-x_{i} \leq 0 & \text { for all } e=\{i, j\} \in E \\
x_{i}+x_{j}-y_{e} \leq 1 & \text { for all } e=\{i, j\} \in E \\
x_{i} \in\{0,1\} & \text { for all } i \in V \\
y_{e} \geq 0 & \text { for all } e \in E
\end{array}
$$

Note that the constraints (3)-(6), in fact, imply the seemingly stronger integrality property $y_{e} \in\{0,1\}$ for all $e \in E$. Also observe that (2) becomes redundant if $b=n$.

We denote the convex hull of the feasible solutions by

$$
C P_{b}^{n}=\operatorname{conv}\left\{(x, y) \in \mathbb{R}^{n(n+1) / 2} \mid(x, y) \text { satisfies }(2)-(6)\right\}
$$

and call it the $b$-clique polytope.

It is easy to see that the $b$-clique polytope is full-dimensional if $b \geq 2$, i.e.,

$$
\operatorname{dim} C P_{b}^{n}=n(n+1) / 2 .
$$

Consequently, each facet-defining inequality for $C P_{b}^{n}$ is unique up to scalar multiplication. Because $C P_{b-1}^{n} \subseteq C P_{b}^{n}$, each inequality that is valid for $C P_{b}^{n}$ is also valid for $C P_{b-1}^{n}$. Moreover, if a valid inequality for $C P_{b}^{n}$ is facet-defining for $C P_{b-1}^{n}$, it is also facet-defining for $C P_{b}^{n}$. 
Remark 2.1. It is interesting to observe that the (valid) inequalities $x_{i} \geq 0$ and $x_{i} \leq 1$ are not facet-defining for $C P_{b}^{n}$ whenever $n \geq 2$ as they are implied by the constraints (3), (4), and (6).

Similarly, inequality (2) is not facet-defining. The vertices $(x, y)$ of $C P_{b}^{n}$ corresponding to cliques of size $b$ satisfy the two independent equations

$$
\sum_{i \in V} x_{i}=b \text { and } \sum_{e \in E} y_{e}=b(b-1) / 2
$$

and thus cannot generate a hyperplane. (See also Lemma 3.1 below).

The $b$-clique polytope is closely related to the Boolean quadric polytope and the quadratic knapsack polytope. Other polytopes related to the $b$ clique polytope are the clique partitioning polytope (Grötschel and Wakabayashi [1990]) and the cut polytope, which arises from the max-cut problem (see, e.g., Barahona and Mahjoub or Deza and Laurent [1992a,b]).

To make the relationship clearer, we present an equivalent formulation of the maximal edge-weighted $b$-clique problem. Let the $(n \times n)$-matrix $D=\left(d_{i j}\right)$ be given and consider the quadratic optimization problem

$$
\max \sum_{i, j \in V} d_{i j} x_{i} x_{j}
$$

subject to the constraints (2) and (5). Because $x_{i}^{2}=x_{i}$ and $y_{\{i, j\}}:=x_{i} x_{j} \geq 0$ holds, the quadratic problem (8) is equivalent to the linear problem (1) with parameters $d_{i}:=d_{i i}$ and $c_{\{i, j\}}:=d_{i j}+d_{j i}, i \neq j$.

For the rest of the paper, we introduce the following notation. Given a vector $(x, y) \in \mathbb{R}^{n(n+1) / 2}$ and subset $S \subseteq V$, we denote by $E(S)$ the set of edges with both endpoints in $S$ and let

$$
x(S)=\sum_{i \in S} x_{i} \quad \text { and } \quad y(S)=\sum_{e \in E(S)} y_{e} .
$$

Each $S \subseteq V$ with $|S| \leq b$ corresponds to a feasible solution $\left(x^{S}, y^{S}\right)$ for $\left(W C P_{b}\right)$ in the obvious way. We call $\left(x^{S}, y^{S}\right)$ the incidence vector of $S$ and $x^{S}$ the node incidence vector of $S$.

2.1. Relationship with the Boolean quadric polytope. The Boolean quadric polytope $Q P^{n}$ was introduced by Padberg [1989] in his study of the unconstrained quadratic zero-one programming problem. In our notation, we have

$$
Q P^{n}=C P_{n}^{n},
$$

i.e., the Boolean quadric polytope is exactly the weighted $b$-clique polytope with parameter $b=n$. 
Every valid inequality for $Q P^{n}$ is, of course, valid for $C P_{b}^{n}$ for any $b \leq$ $n$. However, not all facet-defining inequalities of $Q P^{n}$ also define facets of $C P_{b}^{n}$. Nevertheless, interesting special cases enjoying this property exist. Examples are the "trivial" inequalities mentioned in the following result due to Padberg [1989] and Park et al. [1996].

Proposition 2.1. Let $n \geq 3$ and $b \geq 2$. Then for all $e=\{i, j\} \in E$ :

(i) The inequality $y_{e} \geq 0$ defines a facet of $C P_{b}^{n}$.

(ii) The inequalities $y_{e}-x_{i} \leq 0$ and $x_{i}+x_{j}-y_{e} \leq 1$ define facets of $C P_{b}^{n}$ if and only if $b \geq 3$.

The following proposition exhibits two more classes of inequalities that are facet-defining for both $Q P^{n}$ and $C P_{b}^{n}$.

\section{Proposition 2.2.}

(i) For any $S \subseteq V$ with $|S| \geq 1$ and $T \subseteq V-S$ with $|T| \geq 2$, the cut inequality

$$
\sum_{i \in S, j \in T} y_{\{i, j\}}-y(S)-y(T)-x(S) \leq 0
$$

defines a facet of $C P_{b}^{n}$ if and only if either $|S|=1$ and $b \geq 3$ or $|S| \geq 2$ and $b \geq 4$.

(ii) For any $S \subseteq V$ with $|S| \geq 3$ and integer $\alpha, 1 \leq \alpha \leq|S|-2$, the clique inequality

$$
\alpha x(S)-y(S) \leq \frac{\alpha(\alpha+1)}{2}
$$

defines a facet of $C P_{b}^{n}$ if and only if either $\alpha \leq b-2$ or $S=V$ and $\alpha \leq b-1$.

Remark 2.2. The fact that the inequalities in Proposition 2.2 are facetdefining for $C P_{b}^{n}$ was already observed by Park et al.[1996]. However, the statement and proof concerning the clique inequalities is not quite correct there. It is claimed that for every $1 \leq \alpha \leq|S|-2$ inequality (12) with $S=V$ defines a facet, which is not true if $\alpha \geq b$ (cf. Hunting [1998]).

Recently, Macambira and De Souza [1996] presented the following class of facet-defining inequalities for $C P_{b}^{n}$ that generalizes the cut inequalities. 
Proposition 2.3. Let $S \subseteq V$ with $|S| \geq 1$ and $T \subseteq V-S$ with $|T| \geq 2$ be two disjoint subsets of nodes. For nonnegative integers $\alpha$ and $\beta$ such that $\alpha-\beta=1$, and $|T| \geq \alpha+1$, the $(\alpha, \beta)$-inequality

$$
\sum_{i \in S, j \in T} y_{\{i, j\}}-y(S)-y(T)-\alpha x(S)+\beta x(T) \leq \frac{1}{2} \alpha \beta
$$

defines a facet of $C P_{b}^{n}$ if $\alpha \leq b-4$.

Macambira and De Souza furthermore proved that the $(\alpha, \beta)$-inequalities define facets of $Q P^{n}$. Their result generalizes a result of Padberg [1989] for the so-called generalized cut-inequalities. We will derive a class of facetdefining inequalities that generalizes the clique inequalities in Section 3.

2.2. Relationship with the quadratic knapsack polytope. Given a vector $w=\left(w_{1}, \ldots, w_{n}\right) \in \mathbb{R}^{n}$, we define the quadratic knapsack polytope $Q K P_{b}^{n}(w)$ as the convex hull of those vectors $(x, y) \in \mathbb{R}^{n(n+1) / 2}$ satisfying the conditions (3)-(6) together with the following knapsack generalization of (2):

$$
\sum_{i \in V} w_{i} x_{i} \leq b .
$$

The linear optimization problem over $Q K P_{b}^{n}(w)$ generalizes the classical knapsack problem to quadratic objective functions. This general problem occurs, for example, as a subproblem in the work of Johnson et al..

Letting $Q K P_{b}^{n}$ denote the knapsack polytope $Q K P_{b}^{n}(w)$ in the special case $w=(1,1, \ldots, 1)$, i.e., with (2) for (14), we obtain the weighted $b$-clique polytope as $C P_{b}^{n}=Q K P_{b}^{n}$. .

The two classes of inequalities mentioned in the following proposition are facet-defining for $Q K P_{b}^{n}$ (see Johnson et al. [1993] and Park et al. [1996]).

\section{Proposition 2.4.}

(i) For any node $i \in V$ the star inequality

$$
\sum_{e \ni i} y_{e}-(b-1) x_{i} \leq 0
$$

defines a facet of $C P_{b}^{n}$ if and only if $b \leq n-1$.

(ii) Let $T$ be a sub-tree of $K_{n}$ with set of nodes $V(T),|V(T)|=b+1$, and set of edges $E(T),|E(T)|=b$. Let $3 \leq b \leq n-1$. Then the tree inequality

$$
\sum_{e \in E(T)} y_{e}-\sum_{i \in V(T)}\left(\delta_{i}-1\right) x_{i} \leq 0
$$

where $\delta_{i}$ is the degree of node $i$ in $T$, defines a facet of $C P_{b}^{n}$ if and only if $T$ is not a star or $b=n-1$. 
Tree inequalities were also considered by Macambira and De Souza [1996]. They combined a tree inequality with an $(\alpha, \beta)$-inequality for $\alpha=2$ and $\beta=1$, and showed that the resulting inequality is facet-defining if the underlying tree is a path. Since the resulting inequalities do not seem to be very useful in practice, we do not present them in detail here. We mention that the separation problem for the tree inequalities is $N P$-complete (Hunting [1998]).

\section{New facets For the $b$-Clique POLytope}

We say that the collection $\mathcal{C}=\left\{C_{1}, \ldots, C_{m}\right\}$ of nodes of $K_{n}$ is a generating system of cliques if the associated clique incidence vectors $\left(x^{C}, y^{C}\right)$, $C \in \mathcal{C}$, generate an (affine) hyperplane in $\mathbb{R}^{n(n+1) / 2}$.

If $\mathcal{C}$ is a generating system of cliques, there exists a vector $a \neq 0$ and a number $a_{0}$ such that

$$
a\left(x^{C}, y^{C}\right)=a_{0} \text { for all } C \in \mathcal{C} .
$$

Moreover, up to scalar multiplication, $\left(a, a_{0}\right)$ is uniquely determined by $\mathcal{C}$. We say in this case that $a(x, y)=a_{0}$ is a feasible equality for the generating system $\mathcal{C}$.

Let $a(x, y)=a_{0}$ be a linear equality. Restricting the vector $a$ to the components corresponding to $x$ and to $y$ respectively, let us write the equality $a(x, y)=a_{0}$ as

Then we observe

$$
a^{(1)} x+a^{(2)} y=a_{0} \text {. }
$$

Lemma 3.1. Assume $n \geq 2$, and let $a^{(1)} x+a^{(2)} y=a_{0}$ be a feasible equality for the generating system $\mathcal{C}$ of cliques. Then $a^{(2)} \neq 0$.

Proof. Suppose $a^{(2)}=0$. We will derive a contradiction.

Notice that $a^{(1)} \neq 0$ must hold (otherwise $a=0$ would contradict our assumption that $a(x, y)=a_{0}$ is a feasible equality). Suppose that $a^{(1)}$ has exactly one non-zero coefficient $a_{1}^{(1)}$, say. By scalar multiplication, we may assume $a^{(1)}=1$.

Then the equality $a(x, y)=a_{0}$ can only be $x_{1}=1$ or $x_{1}=0$. In the case $x_{1}=1$, however, the cliques in $\mathcal{C}$ also satisfy the equality $x_{2}-y_{\{12\}}=0$. Hence, the incidence vectors $\left(x^{C}, y^{C}\right), C \in \mathcal{C}$, lie in the intersection of two distinct hyperplanes and, therefore, could not generate a hyperplane. Similarly, in the case $x_{1}=0$, the cliques $C \in \mathcal{C}$ would also satisfy the equality $y_{\{12\}}=0$ and thus could not generate a hyperplane. So we conclude that $a^{(1)}$ has at least two non-zero coefficients $a_{1}^{(1)}$ and $a_{2}^{(1)}$, say. 
Every incidence vector $\left(x^{C}, y^{C}\right), C \in \mathcal{C}$, satisfies $a\left(x^{C}, y^{C}\right)=a^{(1)}\left(x^{C}\right)=$ $\sum_{i=1}^{n} a_{i}^{(1)} x_{i}^{C}=a_{0}$. Squaring this equality, we obtain

$$
\sum_{i=1}^{n}\left[a_{i}^{(1)}\right]^{2}\left[x_{i}^{C}\right]^{2}+\sum_{i \neq j} a_{i}^{(1)} a_{j}^{(1)} x_{i}^{C} x_{j}^{C}=a_{0}^{2} .
$$

Because $\left[x_{i}^{C}\right]^{2}=x_{i}^{C}$ and $x_{i}^{C} x_{j}^{C}=y_{\{i, j\}}^{C}$, we see that the vector $\left(x^{C}, y^{C}\right)$ also satisfies the linear equation

$$
\sum_{i \in V}\left[a_{i}^{(1)}\right]^{2} x_{i}+\sum_{\{i, j\} \in E} 2 a_{i}^{(1)} a_{j}^{(1)} y_{\{i, j\}}=a_{0}^{2} .
$$

Since $a_{1}^{(1)} a_{2}^{(1)} \neq 0$, the latter equation is independent from $a(x, y)=a_{0}$, which implies that the clique incidence vectors lie in the intersection of two distinct hyperplanes, a contradiction to our assumption that $\mathcal{C}$ is a generating system of cliques.

Lemma 3.2. Assume $n \geq 2$, and let $\mathcal{C}$ be a generating system of cliques with associated incidence vectors $\left(x^{C}, y^{C}\right), C \in \mathcal{C}$. Then the projected node incidence vectors $x^{C}, C \in \mathcal{C}$, generate $\mathbb{R}^{n}$.

Proof. If the Lemma were false, the vectors $x^{C}$ would be contained in a hyperplane of $\mathbb{R}^{n}$, i.e., we could find a vector $a^{(1)} \neq 0$ and a number $a_{0}$ such that $a^{(1)} x^{C}=a_{0}$ for all $C \in \mathcal{C}$. With $a=\left(a^{(1)}, 0\right)$, we see that the generating system $\mathcal{C}$ would thus satisfy the equality $a(x, y)=a_{0}$, which contradicts Lemma 3.1.

Let $\mathcal{C}=\left\{C_{1}, \ldots, C_{n+1}, C_{n+2}, \ldots, C_{m}\right\}$ be a generating system of cliques of $K_{n}$. In view of Lemma 3.2, we may assume that the node incidence vectors $x^{C_{1}}, \ldots, x^{C_{n+1}}$ are affinely independent. We say that $\mathcal{C}$ is $b$-strong if $\left|C_{1}\right| \leq \ldots \leq\left|C_{n+1}\right| \leq b-1$ holds.

Note that $\left|C_{1}\right| \leq b-2$ must hold if $\mathcal{C}$ is $b$-strong: otherwise, the $n+1$ vectors $x^{C_{k}}, 1 \leq k \leq n+1$, would satisfy the linear equality $\sum_{i \in V} x_{i}=b-1$ and hence could not be affinely independent.

With the generating system $\mathcal{C}$ of cliques of $K_{n}$ we associate its canonical extension $\overline{\mathcal{C}}$ relative to $K_{N}, N>n$, as follows. $\overline{\mathcal{C}}$ consists of the following collections of subsets of nodes of $K_{N}$ :

$$
\begin{array}{ll}
\mathcal{S}^{0} & :=\{C \mid C \in \mathcal{C}\} \\
\mathcal{S}^{l} & :=\left\{C_{k} \cup\{l\} \mid k=1, \ldots, n+1\right\} \\
\mathcal{T} & :=\left\{C_{1} \cup\{l, s\} \mid n+1 \leq l<s \leq N\right\}
\end{array} \quad(\text { for } l=n+1, \ldots, N)
$$

(Note that $\mathcal{T}=\emptyset$ if $N=n+1$ and that the cardinality of each clique in $\mathcal{S}^{l}$ and $\mathcal{T}$ is bounded by $b$ if $\mathcal{C}$ is $b$-strong). 
Theorem 3.1. ("Lifting Theorem"). If $n \geq 2$ and $\mathcal{C}$ is a generating system relative to $K_{n}$, then the canonical extension $\overline{\mathcal{C}}$ is a generating system relative to $K_{N}$.

Proof. Let $\mathcal{C}=\left\{C_{1}, \ldots, C_{m}\right\}$. The canonical extension $\overline{\mathcal{C}}$ of $\mathcal{C}$ cannot generate all of $\mathbb{R}^{N(N+1) / 2}$ since the feasible equation $a(x, y)=a_{0}$ for $\mathcal{C}$ yields an equality satisfied by $\overline{\mathcal{C}}$ in an obvious way ( $c f$. the "canonical extension" of an inequality below).

To see that $\overline{\mathcal{C}}$ does indeed generate a hyperplane, there is no loss of generality when we assume that the associated incidence vectors $\left(x^{C}, y^{C}\right), C \in \mathcal{C}$, are affinely independent. So $m=n(n+1) / 2$ and hence $|\overline{\mathcal{C}}|=N(N+1) / 2$. It then suffices to show that the clique incidence vectors associated with $\overline{\mathcal{C}}$ are affinely independent.

The incidence vectors corresponding to cliques in $\mathcal{T}$ are independent of all the others (due to their unique 1-entry in the $y$-coordinate $(l, s)$ ). Thus, suppose that there exists some non-trivial affine relation among the other incidence vectors:

$$
\sum_{j=1}^{n(n+1) / 2} \lambda_{j}\left(x^{C_{j}}, y^{C_{j}}\right)+\sum_{l=n+1}^{N} \sum_{k=1}^{n+1} \mu_{l k}\left(x^{C_{k} \cup\{l\}}, y^{C_{k} \cup\{l\}}\right)=0
$$

with $\sum \lambda_{j}+\sum \mu_{l k}=0$.

By assumption, the incidence vectors corresponding to $\mathcal{S}^{0}$ are affinely independent. Hence we must have $\mu_{l k} \neq 0$ for some $l$ and $k$. Considering the $x$-coordinate corresponding to node $l$, we conclude $\sum_{k} \mu_{l k}=0$.

Moreover, considering the $y$-coordinates corresponding to edges joining $l$ to a node in $C_{k}, k=1, \ldots, n+1$, we note that these are in one-toone correspondence with the node incidence vector of $C_{k}$. So the above relation actually implies an affine relation among the node incidence vectors of $C_{1}, \ldots, C_{n+1}$, which contradicts Lemma 3.2 and our labeling of $\mathcal{C}$.

We want to generalize Padberg's [1989] concept of a canonical extension of a valid inequality.

If $a(x, y) \leq a_{0}$ is a valid inequality for $C P_{b}^{n}$, we define its canonical extension $a^{*}(x, y) \leq a_{0}$ to $K_{N}$ by

$$
\begin{array}{llll}
a_{i}^{*}=a_{i} & \forall i \in V, & a_{i}^{*}=0 & \forall i \in V^{\prime} \backslash V \\
a_{e}^{*}=a_{e} & \forall e \in E(V), & a_{e}^{*}=0 & \forall e \in E\left(V^{\prime}\right) \backslash E(V),
\end{array}
$$

where $V=\{1, \ldots, n\}$ and $V^{\prime}=\{1, \ldots, N\}$. Trivially, the canonical extension of a valid inequality for $C P_{b}^{n}$ is valid for $C P_{b}^{N}$. 
If $a(x, y) \leq a_{0}$ is facet-defining for $C P_{b}^{n}$, then the system $\mathcal{C}$ of all cliques $C$ such that $|C| \leq b$ and $a\left(x^{C}, y^{C}\right)=a_{0}$ is a generating system of cliques. We say that $a(x, y) \leq a_{0}$ is $b$-strong if the associated generating system $\mathcal{C}$ is $b$-strong.

Corollary 3.1. Let $a(x, y) \leq a_{0}$ be a b-strong facet-defining inequality for $C P_{b}^{n}, n \geq 2$. Then its canonical extension is facet-defining for any $C P_{b}^{N}$ with $N>n$.

Proof. If $\mathcal{C}$ is the generating system of cliques associated with the facetdefining inequality $a(x, y) \leq a_{0}$, then the incidence vectors of the cliques $\bar{C}$ in the canonical extension $\overline{\mathcal{C}}$ of $\mathcal{C}$ obviously satisfy the equality $a^{*}\left(x^{\bar{C}}, y^{\bar{C}} x\right)=$ $a_{0}$. So $a^{*}(x, y) \leq a_{0}$ must be facet-defining for $C P_{b}^{N}$.

Corollary 3.2. (Padberg [1989]). If $a(x, y) \leq a_{0}$ is a facet-defining inequality for the Boolean quadric polytope $Q P^{n}$, then its canonical extension $a^{*}(x, y) \leq a_{0}$ is facet-defining for $Q P^{n+1}$.

Proof. $a^{*}(x, y) \leq a_{0}$ is valid for $Q P^{n+1}=C P_{n+1}^{n+1}$. By Theorem 3.1, it must be facet-defining.

3.1. Reformulation-Linearization Technique. A useful technique for finding new valid inequalities of a polytope corresponding to an integer zero-one programming problem is the so-called Reformulation-Linearization Technique (RLT) as introduced by Sherali and Adams [1990]. (This work is related to Lovász and Schrijver [1991].)

The RLT consists of two steps, namely a reformulation step and a linearization step. In the reformulation step problem constraints are multiplied with $d$-degree polynomial factors composed of the $n$ binary variables (in our case $\left.x_{1}, \ldots, x_{n}\right)$ and their complements, for some fixed $d \in\{0,1, \ldots, n\}$. The resulting nonlinear program is then linearized by introducing new variables. For our purposes, we use only degree-1 polynomial factors.

As an example, consider the inequality (2)

$$
\sum_{j \in V} x_{j} \leq b
$$

which is not facet-defining ( $c f$. Lemma 3.1). Multiplication by $\left(1-x_{i}\right)$, for some fixed $i \in V$, yields

$$
(b-1) x_{i}+\sum_{j \in V} x_{j}-\sum_{e \ni i} y_{e} \leq b,
$$

which is easily seen to be facet-defining. (We again employ the relations $x_{i}^{2}=x_{i}$ and $x_{i} x_{j}=y_{\{i, j\}}$ for the binary variables $x_{i}$ and $x_{j}$ ). 
As a second example, consider the clique inequality

$$
\alpha x(V)-y(V) \leq \frac{\alpha(\alpha+1)}{2}
$$

for some integer $1 \leq \alpha \leq b-1$. Multiplying (17) with $x_{i}$, we obtain

$$
\alpha x_{i}+\alpha \sum_{e \ni i} y_{e}-x_{i} \sum_{f \in E} y_{f} \leq \frac{\alpha(\alpha+1)}{2} x_{i} .
$$

Instead of introducing new variables $w_{i f}=x_{i} y_{f}$, we just use the relation $x_{i} y_{f} \leq y_{f}$ in order to obtain the $i$-clique inequality

$$
\alpha \sum_{e \ni i} y_{e}-y(V)-\frac{\alpha(\alpha-1)}{2} x_{i} \leq 0 .
$$

The way we derived (18) from (17) implies that (18) is a valid inequality for $C P_{b}^{n}$. (This can also be verified directly).

Theorem 3.2. For every integer $\alpha, 2 \leq \alpha \leq b-1 \leq n-2$, the $i$-clique inequality (18) is facet-defining for $C P_{b}^{n}$.

Proof. We will exhibit a set of $n(n+1) / 2$ affinely independent elements of $C P_{b}^{n}$ satisfying a given $i$-clique inequality with equality.

Consider the incidence vectors $\left(x^{C}, y^{C}\right)$ for $C=\emptyset, C=\{j\}, j \neq i$, and all subsets $C \subseteq V$ with $i \in C$ and $|C| \in\{\alpha, \alpha+1\}$.

It is straightforward to check that all of these incidence vectors satisfy (18) with equality. We leave it to the reader to verify that these incidence vectors also span a subspace of co-dimension 1. (Because $\alpha \leq n-2$, there are "sufficiently many" candidate sets $C$ ).

The $i$-clique inequalities are obviously $b$-strong. So we can apply Corollary 3.1 in the case $n=b+1$ and obtain

Corollary 3.3. Let $S \subseteq V$ and $2 \leq \alpha \leq \min \{b-1,|S|-2\}$. Then the "lifted $i$-clique inequality"

$$
\alpha \sum_{j \in S \backslash i} y_{\{i, j\}}-y(S)-\frac{\alpha(\alpha-1)}{2} x_{i} \leq 0
$$

is facet-defining for $C P_{b}^{n}$.

We refer the interested reader to Hunting [1998] for other classes of facetinducing inequalities. Applying the technique of "sequential lifting" ( $c f$. Wolsey [1975]), one can, for example derive the following class of generalized clique inequalities: 


$$
\alpha\left[x(S)-\sum_{i \in S, j \in T} y_{\{i, j\}}\right]-y(S) \leq \frac{\alpha(\alpha+1)}{2}[1-x(T)+y(T)]
$$

for $S \subseteq V, T \subseteq V \backslash S$ and $1 \leq \alpha \leq|S|-2$.

\section{LAGRANGIAN RELAXATION}

We now assume in our model for the weighted clique problem that we have a complete directed graph $\vec{K}_{n}=(V, A)$ that differs from $K_{n}$ in that each edge $e=\{i, j\} \in E$ is split into two arcs: $(i, j)$ and $(j, i)$, both in $A$. We assume that each arc in $\vec{K}_{n}$ has a weight that equals half the weight of the corresponding edge in $K_{n}$.

For each $\operatorname{arc}(i, j)$ in $\vec{K}_{n}$, we define a $(0,1)$-variable $\vec{y}_{i j}$ that takes the value 1 if the arc $(i, j)$ is in the clique, and value 0 otherwise. With these new variables, $\left(W C P_{b}\right)$ can be transformed into the problem

$$
\max \sum_{i=1}^{n} d_{i} x_{i}+\sum_{(i, j) \in A} \frac{c_{i j}}{2} \vec{y}_{i j}
$$

subject to

$$
\begin{array}{ll}
x_{i}+x_{j}-\vec{y}_{i j} \leq 1, & 1 \leq i<j \leq n \\
\vec{y}_{i j}=\vec{y}_{j i} & 1 \leq i<j \leq n \\
\vec{y}_{i j}-x_{i} \leq 0, & 1 \leq i \neq j \leq n
\end{array}
$$

The star inequalities for $2 \leq \alpha \leq b-1$ :

$$
\sum_{\substack{j=1 \\ j \neq i}}^{n} \vec{y}_{i j}-(b-1) x_{i} \leq 0, \quad 1 \leq i \leq n
$$

The clique inequalities for $S=V, 1 \leq \alpha \leq b-1$ :

$$
\alpha \sum_{i=1}^{n} x_{i}-\frac{1}{2} \sum_{(i, j) \in A} \vec{y}_{i j} \leq \frac{\alpha(\alpha+1)}{2},
$$

The $i$-clique inequalities for $1 \leq i \leq n$ and $2 \leq \alpha \leq b-1$ :

$$
\alpha \sum_{\substack{j=1 \\ j \neq i}}^{n} \vec{y}_{i j}-\frac{1}{2} \sum_{(j, k) \in A} \vec{y}_{j k}-\frac{\alpha(\alpha-1)}{2} x_{i} \leq 0
$$




$$
\begin{array}{ll}
x_{i} \in\{0,1\}, & 1 \leq i \leq n \\
\vec{y}_{i j} \in\{0,1\}, & 1 \leq i \neq j \leq n .
\end{array}
$$

When we now dualize the (in)equalities (20) and (21) by using Lagrangian multipliers $u \geq 0$ and $w$, we obtain the Lagrangian problem $\left(L_{u, w}\right)$ :

$$
\begin{aligned}
\max & \sum_{i=1}^{n} d_{i} x_{i}+\sum_{(i, j) \in A} \frac{c_{i j}}{2} \vec{y}_{i j} \\
& +\sum_{(i, j) \in A} u_{i j}\left(1-x_{i}-x_{j}+\vec{y}_{i j}\right)+\sum_{(i, j) \in A} w_{i j}\left(\vec{y}_{j i}-\vec{y}_{i j}\right)
\end{aligned}
$$

subject to $(22)-(27)$.

An optimal solution of the Lagrangian relaxation $\left(L_{u, w}\right)$ can be efficiently computed as a consequence of the following observation.

Lemma 4.1. A (0,1)-vector $(x, \vec{y})$ satisfies the constraints (22) - (27) if and only if there exists an integer $p \in\{0,1, \ldots, b\}$ such that

$$
\begin{gathered}
\sum_{i=1}^{n} x_{i}=p \\
\sum_{j \in V \backslash i} \vec{y}_{i j}= \begin{cases}p-1 & \text { if } x_{i}=1 \\
0 & \text { otherwise. }\end{cases}
\end{gathered}
$$

Proof. Assume that the $(0,1)$-vector $(x, \vec{y})$ satisfies $(29)$ and $(30)$. Then the conditions (22), (23), (26) and (27) are clearly satisfied. Inequality (24) is equivalent with

$$
(\alpha-p)(\alpha-p+1) \geq 0,
$$

which holds because $\alpha$ and $p$ are integers. Inequality (25) follows in the same way.

Conversely, assume that the $(0,1)$-vector $(x, \vec{y})$ satisfies $(22)-(27)$. Adding all the star inequalities (23) and twice the clique inequality (24) with $\alpha=$ $b-1$, we deduce $\sum x_{i} \leq b$. Let $p:=\sum x_{i}$.

If $p=0$, then (30) is an immediate consequence of (22). If $p=b$, then (23) implies that " $\leq$ " holds in (30). On the other hand, we see from (24) with $\alpha=b-1$ that $\sum_{i, j} \vec{y}_{i j} \geq b(b-1)$ must hold. So (30)) is satisfied with equality.

Finally, consider the case $1 \leq p \leq b-1$. When we add all $i$-clique inequalities (25) with $x_{i}=1$ for $\alpha=p$ (choose $\alpha=2$ in the case $p=1$ ), we 
obtain

$$
\sum_{i, j} \vec{y}_{i j} \leq p(p-1)
$$

On the other hand, (24) with $\alpha=p$ shows

$$
\sum_{i, j} \vec{y}_{i j} \geq p(p-1) .
$$

Hence equality holds. Substitution of this equality into (25) with $\alpha=p$ now yields the desired result.

In view of Lemma 4.1, we can solve problem $\left(L_{u, w}\right)$ in a straightforward way in time $O\left(n^{2} \log n\right)$ :

Note that the objective function of $\left(L_{u, w}\right)$ can the written as

$$
\max \sum_{i \in V} \tilde{d}_{i} x_{i}+\sum_{(i, j) \in A} \tilde{c}_{i j} \vec{y}_{i j} .
$$

Thus, for $p=0,1, \ldots, b$, we (greedily) choose the $p$ "best" nodes $i \in V$, i.e., those nodes $i$ for which the weight $\tilde{d}_{i}$ plus the weights $\tilde{c}_{i, j}$ of the corresponding $p$ heaviest arcs $(i, j)$ is as large as possible). We finally compare these $b+1$ candidate solutions and we take the one with the best objective function value.

4.1. Improving the Lagrangian Relaxation. Let $L(u, w)$ denote the optimal value of the Lagrangian problem $\left(L_{u, w}\right)$ with respect to the objective (28). Since $L(u, w)$ is an upper bound on the objective function value of our integer program $\left(W C P_{b}\right)$ of Section 2 , we are interested in solving the problem

$$
\min \{L(u, w) \mid u \geq 0, w\}
$$

in order to obtain good bounds for a branch-and-bound algorithm (see also Section 5).

One could theoretically improve the Lagrangian bound by dualizing all of the inequalities known to be facet-generating for the polytope $C P_{b}^{n}$. Typically, however, there are too many facet-generating inequalities to make this procedure computationally feasible in practice. So we want to dualize a valid inequality only when it has been found to be violated by the current Lagrangian solution. This implies that we need an efficient algorithm to detect violated inequalities. As an illustration, we give such a separation algorithm for the tree inequalities ( $c f$. Proposition 2.4). We expect that, e.g., the clique and cut inequalities can be treated similarly.

It is $N P$-hard to find a tree inequality that is violated by a given vector $(x, \vec{y}) \in \mathbb{R}^{n^{2}}$ (Hunting [1998]). We will show, however, that this separation problem can be solved in polynomial time when restricted to $(0,1)$-vectors 
$(x, \vec{y})$ satisfying inequalities (29) and (30). This means that we can solve the separation problem for tree inequalities with respect to the solutions of our Lagrangian relaxation of $\left(W C P_{b}\right)$.

Let $T$ be a tree in $K_{n}$ with set $V(T)$ of nodes and set $E(T)$ of (undirected) edges. Denoting by $\delta_{i}(T)$ the degree of node $i \in V(T)$ with respect to $T$, we set

$$
\omega(T):=\sum_{\{i, j\} \in E(T)} \frac{1}{2}\left(\vec{y}_{i j}+\vec{y}_{j i}\right)-\sum_{i \in V(T)}\left(\delta_{i}(T)-1\right) x_{i} .
$$

Thus $(x, \vec{y})$ violates the tree inequality associated with the tree $T$ if and only if $\omega(T)>0$.

Proposition 4.1. Assume that the $(0,1)$-vector $(x, \vec{y})$ satisfies the conditions of Lemma 4.1. Then $(x, \vec{y})$ satisfies all tree inequalities if and only if

$$
\vec{y}_{i j} \leq x_{j} \quad 1 \leq i \neq j \leq n .
$$

Proof. Assume that (32) holds for the vector $(x, \vec{y})$ and let $T$ be a tree with $|V(T)|=b+1$ nodes.

Since $\sum_{i} x_{i} \leq b$, there exists a node $r \in V(T)$ with $x_{r}=0$. Starting from this "root" $r$, we can compute $\omega(T)$ recursively by iteratively adding an edge $(i, j)$ to the subtree $T^{\prime}$ of $T$ already constructed. If $T^{\prime \prime}$ denotes this augmented tree with the new node $j$, we observe

$$
\omega\left(T^{\prime \prime}\right)=\omega\left(T^{\prime}\right)+\frac{1}{2}\left(\vec{y}_{i j}+\vec{y}_{j i}\right)-x_{j} .
$$

In view of $(22)$ and (32) we conclude $\left(\vec{y}_{i j}+\vec{y}_{j i}\right) / 2-x_{j} \leq 0$ and therefore obtain inductively

$$
\omega\left(T^{\prime \prime}\right) \leq \omega\left(T^{\prime}\right) \leq 0 .
$$

Conversely, if (32) is violated at some node $r \in V$ (i.e., $x_{r}=0$ and $\vec{y}_{l r}=1$ for some $l \in V$ ), we take $T$ to a star with center $r$ and $l$ as one of its $b$ leaves. Then $\omega(T)>0$ holds.

\section{Computational Results}

We implemented a branch-and-bound algorithm for the edge-weighted clique problem $\left(W C P_{b}\right)$ as follows. At each node of the search tree, we create subproblems by fixing some variable $x_{i}$ to either 0 or 1 . Following an idea of Lucena [1992], we compute upper bounds for the objective function of the subproblems by first (approximately) solving the corresponding Lagrangian dual problem

$$
\text { (D) } \min \{L(u, w) \mid u \geq 0\}
$$


and then adding new violated inequalities to the objective function of the Lagrangian problem. We then solve the Lagrangian dual problem relative to the new objective function and add new violated inequalities. We repeat this process a fixed number of times. In the following, we will refer to an execution of this process (i.e., solving problem $(D)$ and adding new violated inequalities) as a cycle.

In order to keep the number of dualized constraints manageable, we remove in each cycle those constraints from the objective function for which the associated Lagrangian multipliers are almost 0.

We solve the Lagrangian dual $(D)$ via the subgradient method with step sizes either according Held et al. [1974] with

$$
\lambda_{k}=\rho \frac{f\left(x^{k}\right)-\bar{f}}{\left\|\gamma^{k}\right\|},
$$

where $\bar{f}$ is an estimation of the optimal value $f^{*}$, and coefficient $\rho$ satisfies $0<\rho \leq 2$ or according to the convergent series method (see Shor [1968] and Goffin [1977]) with

$$
\lambda_{k}=\lambda_{0} \alpha^{k}
$$

where $0<\alpha<1$, and $\lambda_{0}$ is the initial step size.

Several preliminary tests lead us to an implementation where we use the first rule for solving Lagrangian dual problems associated to node 0 of the search tree and use the convergent series method for search tree nodes at depth $>0$.

As to the parameter setting, we observed that the correct choice of parameters depends on the type of inequalities we add. For example, the "original" Lagrangian dual $(D)$ could be solved with the convergent series method and $\alpha=0.95$, while we needed $\alpha=0.99$ when we added all triangle clique and cut inequalities. Such high values of $\alpha$ (we set $\lambda_{0}=100$ ) lead to a fairly large number of iterations (about 300) of the subgradient method. All of our computational results are obtained with a fixed setting of the parameters, chosen so as to optimize running times for the "hard instances". We noticed that the choice of a different setting could result in a faster solution for the "easy" problems.

Lower bounds are computed with a simple heuristic solution algorithm proposed by Späth [1985a]: we take the clique given a the current solution and perform a sequence of simple exchange steps (exchange a node in the clique with a node outside) until a local maximum is reached. This heuristic performed extremely well: in all of our experiments, we found the global optimum before the first branching, i.e., at depth 0 . 
Macambira and de Souza [1996] tested a branch-and-cut method on a set of instances with $40 \leq n \leq 48$ and $b=n / 2$. Edge weights were generated by using a parameter $k \in\{1,2,3,4,5\}$. Our computational results for these instances are listed below in Table 1 (positive edge weights) and Table 2 (mixed, i.e., positive and negative edge weights).

The column "gap \%" displays the relative difference between the optimal solution and the upper bound computed at depth 0 . The columns "max depth" and "\# branch nodes" refer to the search tree.

The running time resulting from our approach seems to be at least comparable with the branch-and-cut method of Macambira and de Souza [1996]. On the hard instances, in particular, our approach led to an improvement by a factor of 2 or 3 . Our computations were carried out on a HP 9000/735 (125 Mhz), whereas Macambira and de Souza implemented their algorithm on a SUN Sparc 1000 machine, using CPLEX 3.0. 


\begin{tabular}{|c|c|c|c|c|c|c|c|}
\hline$n$ & $k$ & $\begin{array}{l}\text { optimal } \\
\text { value }\end{array}$ & 1st node & $\begin{array}{c}\text { gap } \\
\%\end{array}$ & $\begin{array}{l}\text { max. } \\
\text { depth }\end{array}$ & $\begin{array}{c}\text { \# branch } \\
\text { nodes }\end{array}$ & $\begin{array}{l}\text { time } \\
\text { (sec.) }\end{array}$ \\
\hline \multirow{5}{*}{40} & 1 & 109346 & 109819.2 & $\overline{0.4}$ & 4 & 11 & 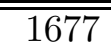 \\
\hline & 2 & 82451 & 82517.1 & 0.1 & 2 & 5 & 946 \\
\hline & 3 & 68759 & 69339.4 & 0.8 & 5 & 23 & 2115 \\
\hline & 4 & 60782 & 61601.3 & 1.3 & 5 & 37 & 2915 \\
\hline & 5 & 60513 & 60561.7 & 0.1 & 1 & 3 & 917 \\
\hline \multirow{5}{*}{42} & $\overline{1}$ & $\overline{\overline{120299}}$ & "121308.0 & $\overline{\overline{0.8}}$ & 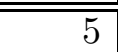 & $\overline{\overline{43}}$ & 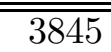 \\
\hline & 2 & 87810 & 88950.6 & 1.3 & 6 & 47 & 4203 \\
\hline & 3 & 76554 & 77081.3 & 0.7 & 11 & 39 & 3947 \\
\hline & 4 & 69482 & 69644.5 & 0.2 & 2 & 7 & 1381 \\
\hline & 5 & 67383 & 67453.5 & 0.1 & 1 & 3 & 1245 \\
\hline \multirow{5}{*}{44} & $\overline{11}$ & $\overline{136525}$ & 136846.5 & $\overline{\overline{0.2}}$ & 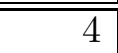 & $\overline{\overline{11}}$ & 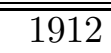 \\
\hline & 2 & 98186 & 99285.3 & 1.1 & 4 & 31 & 3672 \\
\hline & 3 & 84675 & 84856.6 & 0.2 & 2 & 7 & 1332 \\
\hline & 4 & 75274 & 75559.8 & 0.4 & 2 & 7 & 1403 \\
\hline & 5 & 69540 & 69777.6 & 0.3 & 12 & 27 & 4113 \\
\hline \multirow{5}{*}{46} & 1 & $\overline{142985}$ & $\overline{144377.5}$ & $\overline{\overline{1.0}}$ & 7 & $\overline{\overline{57}}$ & $\overline{5590}$ \\
\hline & 2 & 108243 & 109777.5 & 1.4 & 6 & 81 & 7481 \\
\hline & 3 & 94859 & 95213.7 & 0.4 & 4 & 15 & 2421 \\
\hline & 4 & 78747 & 79674.4 & 1.2 & 5 & 41 & 4222 \\
\hline & 5 & 72431 & 73297.8 & 1.2 & 8 & 43 & 4434 \\
\hline \multirow{5}{*}{48} & 1 & $\bar{~} 163397$ & $\overline{164435.4}$ & $\overline{\overline{0.6}}$ & 6 & $\overline{477}$ & $\overline{44959}$ \\
\hline & 2 & 115471 & 118219.7 & 2.4 & 11 & 295 & 27479 \\
\hline & 3 & 96666 & 98305.9 & 1.7 & 8 & 117 & 11166 \\
\hline & 4 & 88728 & 89112.5 & 0.4 & 4 & 11 & 2122 \\
\hline & 5 & 82117 & 82361.6 & 0.3 & 4 & 9 & 2093 \\
\hline
\end{tabular}

TABle 1. Results for Macambira/De Souza instances (positive weights) 


\begin{tabular}{|r|c|c|r|r|r|r|r||}
\hline & & $\begin{array}{c}\text { optimal } \\
\text { value }\end{array}$ & 1st node & $\begin{array}{c}\text { gap } \\
\%\end{array}$ & $\begin{array}{c}\text { max. } \\
\text { depth }\end{array}$ & $\begin{array}{c}\text { \# branch } \\
\text { nodes }\end{array}$ & $\begin{array}{c}\text { time } \\
\text { (sec.) }\end{array}$ \\
\hline \hline \multirow{4}{*}{40} & 1 & 70348 & 73580.6 & 4.6 & 8 & 175 & 14079 \\
& 2 & 45404 & 45743.8 & 0.7 & 5 & 11 & 1857 \\
& 3 & 34091 & 34502.9 & 1.2 & 4 & 9 & 1129 \\
& 4 & 27758 & 28180.3 & 1.5 & 3 & 15 & 2548 \\
& 5 & 27967 & 28076.3 & 0.4 & 5 & 13 & 2785 \\
\hline \hline \multirow{4}{4}{42} & 1 & 81633 & 84997.2 & 4.1 & 9 & 185 & 14841 \\
& 2 & 46828 & 48091.0 & 2.7 & 5 & 43 & 4159 \\
& 3 & 36689 & 37014.2 & 0.9 & 3 & 13 & 2130 \\
& 4 & 35987 & 36211.2 & 0.6 & 3 & 7 & 534 \\
& 5 & 35460 & 35710.3 & 0.7 & 3 & 7 & 1800 \\
\hline \hline \multirow{4}{*}{44} & 1 & 90620 & 94499.8 & 4.3 & 11 & 307 & 27445 \\
& 2 & 56960 & 57978.3 & 1.8 & 5 & 25 & 3329 \\
& 3 & 40697 & 41101.0 & 1.0 & 3 & 9 & 2142 \\
& 4 & 32601 & 33157.9 & 1.7 & 7 & 35 & 4208 \\
& 5 & 29407 & 29639.5 & 0.8 & 3 & 9 & 1502 \\
\hline \hline & 1 & 99550 & 102398.6 & 2.9 & 12 & 101 & 10348 \\
& 2 & 58361 & 59539.5 & 2.0 & 8 & 37 & 4579 \\
46 & 3 & 43915 & 45109.0 & 2.7 & 6 & 49 & 5418 \\
& 4 & 32968 & 34354.0 & 4.2 & 14 & 97 & 10185 \\
& 5 & 31000 & 31252.6 & 0.8 & 3 & 9 & 2350 \\
\hline \hline & 1 & 113478 & 118353.3 & 4.3 & 12 & 539 & 55917 \\
& 2 & 61768 & 65358.1 & 5.8 & 10 & 377 & 36963 \\
48 & 3 & 45941 & 46572.9 & 1.4 & 5 & 23 & 3277 \\
& 4 & 36903 & 37176.3 & 0.7 & 3 & 9 & 2257 \\
& 5 & 31351 & 32067.0 & 2.3 & 5 & 33 & 4505 \\
\hline
\end{tabular}

TABle 2. Results for Macambira/De Souza instances (mixed weights) 
Acknowledgement. We thank Dr. Macambira and Prof. de Souza for making their data sets available to us.

\section{REFERENCES}

[1] F. Barahona and R. Mahjoub [1986]: On the cut polytope. Mathematical Programming $36,157-173$.

[2] F. Barahona, M. Jünger, and G. Reinelt [1989]: Experiments in quadratic 0-1 programming. Mathematical Programming 44, 127-137.

[3] E. Boros and P.L. Hammer [1993]: Cut-polytopes, Boolean quadric polytopes and nonnegative quadratic pseudo-Boolean functions. Mathematics of Operations Research 18, $245-253$.

[4] K.M. Bretthauer, B. Shetty, and S. Syam [1995]: A branch and bound algorithm for integer quadratic knapsack problems. ORSA Journal of Computing 7, 109-116.

[5] P. Chaillou, P. Hansen, and Y. Mahieu [1989]: Best network flow bounds for the quadratic knapsack problem. Springer Lecture Notes in Mathematics 1403, 225-235.

[6] M. Deza and M. Laurent [1992a]: Facets for the cut cone I. Mathematical Programming $56,121-160$.

[7] M. Deza and M. Laurent [1992b]: Facets for the cut cone II: Clique-web inequalities. Mathematical Programming 56, 161-188.

[8] G. Dijkhuizen and U. Faigle [1993]: A cutting-plane approach to the edge-weighted maximal clique problem. European Journal of Operational Research 69, 121-130.

[9] E. Erkut, T. Baptie, and B. von Hohenbalken [1990]: The discrete p-maxian location problem. Computers \& Operations Research and their Application to Problems of World Concern 17, 51-61.

[10] U. Faigle, R. Garbe, K. Heerink, and B. Spieker [1994]: LP-relaxations for the edgeweighted subclique problem. In: Operations Research '93, A. Bachem, U. Derigs, M. Jünger, R. Schrader (eds.), Physica-Verlag, Heidelberg, 157-160.

[11] J.L. Goffin [1977]: On the convergence rates of subgradient optimization methods. Mathematical Programming 13, 329-347.

[12] M. Grötschel and Y. Wakabayashi [1990]: Facets of the clique partitioning polytope. Mathematical Programming 47, 367-387.

[13] J. Hardin, J. Lee, and J. Leung [1995]: On the Boolean-quadric packing uncapacitated facility-location polytope. Technical report December 1995, Department of Mathematics, University of Kentucky.

[14] M. Held, P. Wolfe, and H.P. Crowder [1974]: Validation of subgradient optimization. Mathematical Programming 6, 62-88.

[15] C. Helmberg, F. Rendl, and R. Weismantel [1996]: Quadratic knapsack relaxations using cutting planes and semidefinite programming. Springer Lecture Notes in Computer Science 1084, 175-189.

[16] M. Hunting [1998]: Relaxation Techniques for Discrete Optimization Problems; Theory and Algorithms, Ph.D. Dissertation, University of Twente.

[17] E.L. Johnson, A. Mehrotra, and G.L. Nemhauser [1993]: Min-cut clustering. Mathematical Programming 62, 133-151.

[18] M.J. Kuby [1987]: Programming models for facility dispersion: the p-dispersion and maxisum dispersion problems. Geographical Analysis 9, 315-329.

[19] L. Lovász and A. Schrijver [1991]: Cones of matrices and set-functions and 0-1 optimization. SIAM Journal on Optimization 1, 166-190.

[20] A. Lucena [1992]: Steiner problems in graphs: Lagrangian relaxation and cuttingplanes. COAL Bulletin, Mathematical Programming Society 21, 2-8. 
[21] E.M. Macambira and C.C. de Souza [1996]: The edge-weighted clique problem: valid inequalities, facets and polyhedral computations. Technical report IC-97-14, Instituto de Computação, Universidade Estadual de Campinas, Brazil, 1996.

[22] M A. Mehrotra [1997]: Cardinality constrained Boolean quadratic polytope. Discrete Applied Mathematics 79, 137-154.

[23] G.L. Nemhauser and L.A. Wolsey [1988]: Integer and Combinatorial Optimization. Wiley, New York.

[24] M. Padberg [1989]: The Boolean quadric polytope: some characteristics, facets and relatives. Mathematical Programming 45, 139-172.

[25] K. Park, K. Lee, and S. Park [1996]: An extended formulation approach to the edgeweighted maximal clique problem. European Journal of Operational Research 95, 671-682. [26] S.S. Ravi, D.J. Rosenkrantz, and G.K. Tayi [1994]: Heuristic and special case algorithms for dispersion problems. Operations Research 42, 299-310.

[27] H.D. Sherali and W.P. Adams [1990]: A hierarchy of relaxations between the continuous and convex hull representations for zero-one programming problems. SIAM Journal on Discrete Mathematics 3, 411-430.

[28] H.D. Sherali, Y. Lee, and W.P. Adams [1995]: A simultaneous lifting strategy for identifying new classes of facets for the Boolean quadric polytope. Operations Research Letters 17, 19-27.

[29] N.Z. Shor [1968]: On the rate of convergence of the generalized gradient method. Kibernetika 4, 98-99.

[30] N.Z. Shor [1970]: Convergence rate of the gradient descent method with dilatation of the space. Cybernetics 6, 102-108.

[31] H. Späth [1985]: Heuristically determining cliques of given cardinality and with minimal cost within weighted complete graphs. Zeitschrift für Operations Research 29, 125131.

[32] L.A. Wolsey [1975]: Faces for a linear inequality in 0-1 variables. Mathematical Programming $4,165-178$.

Department of Applied Mathematics, University of Twente, P.O.Box 217, 7500 AE Enschede, The Netherlands

E-mail address: $\{$ faigle,kern\}@math.utwente.nl 